\title{
Virtual Science in Senology: Let Us Not Throw out the Baby with the Bathwater
}

\author{
Nadia Harbeck ${ }^{a}$ Christoph Thomssen ${ }^{b}$ Michael Gnant ${ }^{c}$ \\ a Breast Center, OB\&GYN Department and CCCLMU, LMU University Hospital, Munich, Germany; \\ ${ }^{b}$ Universitätsfrauenklinik, Martin-Luther-Universität Halle-Wittenberg, Halle/Saale, Germany; ${ }^{c}$ Comprehensive \\ Cancer Center, Medical University of Vienna, Vienna, Austria
}

In the June 2019 issue of Breast Care [1] we discussed the necessity of digitalization in medicine in general and for breast cancer management in particular. Certainly, we did not know back then that less than a year later a pandemic would force the international scientific community to digitalize basically overnight.

Undoubtedly, the COVID-19 pandemic has abruptly changed our professional (and private) lives. As a response to the recommendations of social distancing, digitalization of our clinical and scientific workplace has been one of the major changes. As clinicians and scientists, we have all been forced to rapidly exchange the personal contacts we were used to for internet-based virtual communication. Also, and on rather short notice, the world's largest oncology conference, i.e., ASCO 2020, together with other conferences such as ESMO Breast 2020, has been transferred to a virtual format. In the fall of 2020 , ESMO will also provide a completely virtual program.

As of June 2020, we can look back at 3 months of forced digitalization and we feel that the rapid change towards a digitalized senological community holds great promise for the future. Tele- and videoconferences work well and should definitively be utilized more in the future. We have learned that they require rather thorough preparation in order to be most effective. Virtual conferences and symposia have the potential to communicate scientific news rapidly, and to reach more people. They may also be able to attract new groups of attendees - those who are not able to travel easily for personal, political, or professional reasons.
Nevertheless, even though virtual meetings are attractive as they can save unnecessary travel time, they work particularly well with colleagues and teams who already know and trust each other. Therefore, virtual meetings will certainly not be able to fully replace physical meetings in the future. Networking is an important part of clinical science - brilliant practice-changing projects often start with an informal and spontaneous exchange of ideas. Long-term cooperation often begins with casual encounters at major meetings. In particular, young clinician scientists benefit from such networking outside their everyday clinical routine, networking among each other as well as with potential mentors on a national and most importantly also on an international level. While large virtual conferences help to ensure the continuity of presentation of new ground-breaking data, they cannot replace the vibrant and exciting live atmosphere when practice-changing data is presented. Last but not least, we have learned that our colleagues have different preferences when it comes to scientific meetings; some prefer the flexibility of watching the presentations from home, while others prefer to set aside time from a busy clinical schedule to attend a meeting and absorb new data as well as have direct scientific discussions with colleagues.

In clinical medicine and academia, we often tend to be structurally conservative and somewhat resistant to procedural progress. While industry, for example, already places great emphasis on virtual meetings for applications and job interviews, we still prefer personal meetings. In countries such as Germany, Austria, and Switzerland, the rapid approach to digitalization over the last few months 
required a steep learning curve at the cancer centers as sometimes excessively stringent data protection requirements clashed with urgent clinical and scientific needs. There are many more things that we need to set up in the digital world next to webinars and conferences, i.e., teaching and advising, joint study groups, scientific think tanks, etc. Online platforms established for completely other purposes that function well with artificial intelligencebased algorithms can certainly teach us about digital opportunities. In general, it appears that Europe, and academic Europe in particular, is lagging behind in terms of digitalization as compared to other regions of the world, or to industry as mentioned before. Now is the chance to catch up - we do hope that the current crisis will provide the long-needed boost to digitalization both structurally and in terms of equipment and create the necessary receptiveness and creativity in the scientific community.

The rapid digitalization forced upon us by the COVID-19 pandemic now offers the chance to open a more virtual world in clinical sciences. We should embrace the challenge without losing the benefits of personal realworld interactions. We should be more conscious with travel requirements, both for personal as well as for ecological reasons; yet, we should not underestimate the importance of personal real-world interactions for advancing our clinical and scientific community. Thus, let us not throw out the baby with the bathwater by abandoning personal face-to-face contact completely - to get the maximum benefit from scientific exchange, we will need to find a balance between online and offline person-to-person meetings.

\section{Conflict of Interest Statement}

M. Gnant reports personal fees/travel support from Amgen, AstraZeneca, Celgene, EliLilly, Invectys, Pfizer, Novartis, Puma, Nanostring, Roche, Medison, and LifeBrain (all outside the submitted work). An immediate family member is employed by Sandoz.

N. Harbeck reports minority ownership of Westdeutsche Studiengruppe (WSG) as well as honoraria for consulting and/or lectures from Agendia, Amgen, Astra Zeneca, BMS, Celgene, DaiichiSankyo, Genomic Health, Lilly, MSD, Novartis, Odonate, Pierre Fabre, Pfizer, Roche, Samsung, Sandoz/Hexal, and Seattle Genetics (all outside the submitted work).

C. Thomssen is on the advisory board of or has had presentations for: Amgen, AstraZeneca, Celgen, Daiichi-Sankyo, Eisai, Lilly, MSD, Mundipharma, Medapharm, Novartis, Pfizer, PierreFabre, Roche, Tesaro, and Vifor (all outside the submitted work).

\section{Funding Sources}

None.

\section{Author Contributions}

All of the authors contributed equally to the conception of this paper. N. Harbeck wrote the first draft and C. Thomssen and M. Gnant took part in revising it.

\section{References}

1 Harbeck N. Digitalization in Medicine: It Is Our Chance and Responsibility Now to Shape the Digital Future of Breast Cancer Management. Breast Care (Basel). 2019 Jun;14(3):128-9. 